\title{
USO DE FUENTES DOCUMENTALES -IMPRESAS Y DIGITALES- CON FINES ACADÉMICOS ENTRE EL ALUMNADO UNIVERSITARIO
}

\author{
Rubén Comas Forgas; rubencomas@uib.es \\ Jaume Sureda Negre; sureda.negre@gmail.com \\ Tomeu Mut Amengual ; tomeu.mut@uib.es \\ Universidad de las Islas Baleares
}

\section{RESUMEN:}

En este trabajo se presentan los resultados obtenidos en una investigación acerca de la frecuencia de uso de las bibliotecas -universitarias y no universitarias- e Internet entre el alumnado universitario para documentarse. También se describen las principales actividades académicas para las que los universitarios hacen uso de Internet como fuente de información. De los resultados se desprende que Internet monopoliza las búsquedas del alumnado y que la principal actividad para la que hacen uso de la Red es la elaboración de trabajos.

PALABRAS CLAVE: TIC, alfabetización informacional, competencias, bibliotecas.

SUMMARY: In this paper we present the results of an investigation of the frequency of library and Internet use by university students to locate information with academic purposes. It also describes the major academic activities for which the university students make use of Internet as an information source. The results show a monopoly of the Internet as search resource and that the main activity for which uses the Net is the elaboration of essays.

KEYWORDS: ICT, information literacy, competences, libraries. 


\section{INTRODUCCIÓN.}

El advenimiento y adopción extensiva de las TIC ha traído consigo el surgimiento de nuevos entornos, recursos, formatos y métodos de búsqueda, localización y gestión de la información de carácter académico. A consecuencia de estos cambios han surgido nuevas necesidades que ponen en relación el potencial de los recursos con la manera en que son utilizados. Así, han aparecido formas de alfabetización modernas que se han englobado en lo que se conoce como las "alfabetizaciones múltiples" -multiple literacies- o las "nuevas alfabetizaciones" -new literacies- (Coll y Rodríguez, 2008). Dentro de este epígrafe encontramos, por un lado, formas de alfabetización que guardan estrecha relación con las, por así decirlo, "alfabetizaciones tradicionales"; esto es, aquellas ligadas al manejo del alfabeto o códigos comunicativos -lectura, escritura, operaciones matemáticas, etc.-. En segundo lugar, afloran "nuevas" maneras de alfabetización que no guardan relación directa con el carácter tradicional del término y que se centran en el manejo operativo de los recursos y posibilidades que ofrecen las TIC; en este sentido encontramos, entre otros, conceptos como: "alfabetización informática", "alfabetización en TIC", "alfabetización digital", "alfabetización computacional" o "alfabetización informacional" (Bawden, 2002).

Varis (2003), enumera cinco dimensiones o competencias requeridas para poder participar de forma activa, autónoma y eficiente en el vasto espectro de posibilidades que ofrecen las TIC, siendo: Alfabetización tecnológica; Alfabetización informacional; Creatividad mediática; Alfabetización global y Alfabetización responsable.

Nuestro trabajo se enmarca en el segundo de los ámbitos descritos por el profesor finlandés y, con mayor concreción, se ajusta a la capacidad o competencia de recopilar o buscar información. Además, nuestro objetivo se centra en un escenario muy concreto, con unos actores determinados y un conjunto de necesidades singulares: búsqueda de información con fines académicos por parte del alumnado universitario pregraduado.

Existen un relevante número de aproximaciones empíricas al campo de las estrategias de documentación académica por parte del alumnado universitario (Aditaka y Anwar, 2006; Head, 2007; Kuruppu, 2008; Martin et. al, 2009; Zhang, Anghelescu y Yuan, 2005, Vondraceck, 2007). Si nos ceñimos a propuestas que basan su análisis en la utilización de las bibliotecas universitarias como fuentes de documentación destacan, entre otros muchos, los trabajos de: Hiller y Self (2002), quienes describieron la frecuencia de utilización de las bibliotecas de las Universidades de Washington y Virginia entre los años 1998 y 2001; Hayden y colaboradores (2005), midieron la frecuencia de uso, por parte de los alumnos, de los servicios bibliotecarios del Instituto Tecnológico de Waterford; Bridges (2008), trató el uso de la biblioteca de la Universidad del Estado de Oregón con una muestra de 949 alumnos pregraduados; Baer y Li (2009), analizaron las estrategias documentales usadas, en el marco de la biblioteca universitaria, por estudiantes de Ingeniería del Georgia Institute of Technology; Arias y Simón (2008), ya en España, estudiaron los hábitos de uso de las bibliotecas de la Universidad Complutense de Madrid. En cuanto al empleo de fondos de bibliotecas no universitarias para la localización de información con fines académicos entre el alumnado, encontramos datos al respecto en la investigación de Mckenna (2007), en la que se analizó, con una muestra de 1.575 alumnos daneses de Bachillerato y universidad, el uso que éstos hacían de las bibliotecas públicas no universitarias para actividades 
de naturaleza académica; Agosto, Paone e Ipock (2007), describieron las diferencias en cuanto a género en el uso de bibliotecas públicas entre alumnado preuniversitario.

Si fijamos la atención en la literatura centrada en trabajos en los que se describe el uso de Internet como fuente de documentación académica son destacables trabajos como los de: WenHua (2000), quien mediante metodologías cualitativas estudió la autoeficacia en la competencia de búsqueda de información en contextos electrónicos; O’Hanlon (2002), midió a través de una prueba test la competencia para localizar información de los alumnos de la Universidad Estatal de Ohio; Slaouti (2002), en Manchester, mediante un estudio de carácter cuantitativo analizó la aceptación entre el alumnado de Internet como fuente documental; McMartin y colaboradores (2008), mediante un estudio cuantitativo con una muestra de 4.678 universitarios, analizó el uso de recursos digitales con fines académicos; Nwagwu y colaboradores (2009), precisó los factores que favorecen el uso de Internet como herramienta para desarrollar tareas de documentación académica; Marzal y Calzada (2003), ya en España, desarrollaron un trabajo entre alumnado de la Universidad Carlos III sobre necesidades y hábitos informativos de los universitarios cuando usan la Red para documentarse; Sureda y Comas (2006), centraron su análisis en el alumnado de la carrera de Educación Social de la Universidad de las Islas Baleares y, por medio de un cuestionario, estudiaron el uso de Internet como fuente de documentación académica entre dicho colectivo; finalmente, Comas (2009), en su trabajo doctoral sobre el plagio académico entre el alumnado universitario dirige parte de su análisis hacia las fuentes de documentación consultadas por los universitarios con fines académicos.

El objeto del presente trabajo es, en primer lugar, conocer y precisar la frecuencia de utilización de los servicios bibliotecarios -universitarios y no universitarios- e Internet para la búsqueda de información con fines académicos entre el alumnado pregraduado de la UIB y, en segundo término, precisar las actividades académicas para las que realizan búsquedas en Internet.

\section{MÉTODO.}

\subsection{Muestra y población.}

Universidad de las Islas Baleares (UIB) que han sido seleccionados mediante muestreo aleatorio a partir de la población compuesta por el total de alumnado matriculado en estudios de Diplomatura, Ingenierías y Licenciatura de los cursos segundo, tercero, cuarto y quinto: 7.736 alumnos según datos relativos al curso 2007-08 del vicerrectorado de alumnos de la UIB. EI tamaño de la muestra con el que se ha trabajado supone un error muestral de $\pm 3,4 \%$, para los datos totales, estimado para un nivel de confianza del $95 \%$ y bajo la condición más desfavorable de $p=q=0.05$.

La recogida de datos se realizó en el Campus de la UIB durante el mes de Noviembre de 2009 mediante la aplicación individual y anónima de un cuestionario por parte de 4 personas previamente formadas. En la Tabla I se detallan las características de la muestra. 


\subsection{Fuente de datos y variables de estudio.}

Los datos empleados en la confección del presente artículo provienen de la "Encuesta sobre las competencias en Alfabetización Informacional del alumnado de la Universidad de las Islas Baleares", proyecto de investigación financiado por la Fundación IBIT del Gobierno de las Islas Baleares en el marco del Plan Avanza del Ministerio de Industria, Turismo y Comercio de España cuya principal meta se fijaba en conocer el nivel competencial del alumnado pregraduado de la UIB en aspectos relativos a la Alfabetización Informacional. Se establecieron cinco ámbitos de análisis que se ajustan a las cinco competencias básicas descritas y acordadas internacionalmente en el campo de la Alfabetización Informacional (ACRL, 2000): a) Identificación de las necesidades de información; b) Estrategias de búsqueda de información; c) Evaluación y tratamiento de la información; d) Utilización y elaboración de la información y e) Aspectos éticos y sociales. El instrumento de recogida de datos constaba de un total de 41 preguntas de tipo: abiertas, cerradas dicotómicas, cerradas politómicas, de posición y escalas de respuesta.

Las variables de referencia para elaborar este trabajo quedan enmarcadas en los campos competenciales de "Estrategias de búsqueda de información" e "Identificación de las necesidades de información"; concretamente:

- Utilización de servicios bibliotecarios (universitarios y no universitarios) e Internet (motores de búsqueda generalistas, bases de datos académicas, portales especializados y blogs) para buscar información con fines académicos

- Actividades académicas para las que se hace uso de Internet como fuente de información

A continuación, se relacionaron los resultados de las características descriptivas del alumnado participante: edad, género, curso matriculado, área de conocimiento y número de trabajos académicos elaborados durante el curso 2008-09.

Tabla I: Características y distribución de la muestra del estudio (tamaño de la muestra = 739 alumnos).

\begin{tabular}{|l|l|l|}
\hline Variable & N & $\%$ \\
\hline Género: & 305 & $66,9 \%$ \\
Mujer & 151 & $33,1 \%$ \\
Hombre & & \\
\hline Edad: & 109 & $23,9 \%$ \\
Entre 17 y 20 años & 270 & $59,1 \%$ \\
Entre 21 y 25 años & 40 & $8,8 \%$ \\
Entre 26 y 30 años & 38 & $8,3 \%$ \\
Más de 30 años & & \\
\hline Área de conocimiento & 457 & $61,8 \%$ \\
Ciencias Sociales y Jurídicas & 71 & $9,6 \%$ \\
Ciencias de la Salud & 46 & $6,2 \%$ \\
Humanidades & 165 & $22,3 \%$ \\
Ciencias Experimentales & & \\
\hline Curso matriculado: & 117 & $25,6 \%$ \\
\hline Segundo & & \\
\hline
\end{tabular}




\begin{tabular}{|l|l|l|}
\hline Tercero & 214 & $46,8 \%$ \\
Cuarto & 91 & $19,9 \%$ \\
Quinto & 35 & $7,7 \%$ \\
\hline № de trabajos elaborados el curso 2008-09 & & \\
Entre 0 y 5 & 257 & $56,5 \%$ \\
Entre 6 y 10 & 107 & $23,5 \%$ \\
Más de 11 & 91 & $20 \%$ \\
\hline
\end{tabular}

\subsection{Análisis de datos.}

Respecto a cada una de las variables de categoría se ha efectuado el cálculo de la frecuencia y el porcentaje. Acto seguido, con el propósito de poder establecer la potencial asociación entre las variables en base a las que se hace operativa la competencia de estrategias de búsqueda de información académica, sobre las que se elabora este trabajo, y las características del alumnado o variables categóricas (Tabla II), se han diseñado tablas de contingencia para cada una de las variables y se ha procedido a implementar la prueba de chi-cuadrado calculándose el coeficiente de correlación de Pearson de cara a obtener el nivel de significación ${ }^{1}$ de las asociaciones resultantes. En el diseño de las tablas de contingencia se ha efectuado una recodificación de las variables analizadas; así en relación a la frecuencia de utilización de las bibliotecas -universitarias y no universitarias- e Internet para buscar información académica se ha agrupado la muestra en "No usuarios o usuarios infrecuentes" (casos en los que la frecuencia declarada por el alumnado era "Nunca" o "A veces"), "Usuarios medios" (casos en los que la respuesta era "A menudo") y "Usuarios habituales" (casos en los que la frecuencia declarada era "Muy a menudo" o "Siempre"). En cuanto a la frecuencia de uso de Internet para llevar a cabo actividades académicas, la recodificación hecha ha sido: "Frecuencia baja" (casos en los que la frecuencia era "Nunca" o "A veces"), "Frecuencia media" (casos en los que la respuesta era "A menudo") y "Frecuencia alta" (para las frecuencias "Muy a menudo" o "Siempre"). Para todos los análisis generados se he empleado el paquete estadístico "Statistical Package for the Social Sciences" (SPSS versión 15.0).

\section{RESULTADOS.}

\subsection{Frecuencia de utilización del servicio de bibliotecas de la UIB y otras bibliotecas no universitarias para buscar información con fines académicos.}

Como ilustra la Tabla II, la frecuencia de uso de la biblioteca de la UIB es bastante superior a la de otras bibliotecas no universitarias. Un poco más de dos de cada diez universitarios de la UIB $(2,3 \%)$ emplea la biblioteca de la institución siempre que tiene que buscar información académica. A destacar el dato referente a que el $74,4 \%$ de los alumnos de la UIB nunca visitan otras bibliotecas diferentes de la de la universidad para documentarse con fines académicos.

En la Tabla III se presentan los resultados y el valor de la prueba de chi-cuadrado de Pearson para cada una de las características tipo de la muestra y se puede observar que: a) en relación al uso

\footnotetext{
${ }^{1}$ Dado el error muestral de partida de $\pm 3,4 \%$, se ha optado por establecer el nivel de significación (en la prueba de correlación de Pearson) en 0,01 para mejorar el rigor de la explotación de los datos y la generación de conclusiones.
} 
de la biblioteca de la UIB: se da una asociación fuerte entre la frecuencia de utilización y el curso en que están matriculados y el área de conocimiento; b) relativa al uso de otras bibliotecas: existe significación entre la frecuencia de uso y el área de conocimiento.

Tabla II: Frecuencia de utilización de la biblioteca de la UIB y de otras bibliotecas no universitarias para buscar información académica entre el alumnado a lo largo del curso 2008-09 ( $N=739$ ).

\begin{tabular}{|l|l|l|}
\hline $\begin{array}{l}\text { Frecuencia } \\
\text { utilización }\end{array}$ & $\begin{array}{l}\text { Biblioteca } \\
\text { de la UIB }\end{array}$ & $\begin{array}{l}\text { Bibliotecas no } \\
\text { universitarias }\end{array}$ \\
\hline Nunca & $36,1 \%$ & $74,4 \%$ \\
\hline A veces & $37,8 \%$ & $18,3 \%$ \\
\hline A menudo & $14,5 \%$ & $2,8 \%$ \\
\hline Muy a menudo & $6,9 \%$ & $0,3 \%$ \\
\hline Siempre & $2,3 \%$ & $0,4 \%$ \\
\hline No sabe o No contesta & $2,4 \%$ & $3,8 \%$ \\
\hline Total & $100 \%$ & $100 \%$ \\
\hline
\end{tabular}

Tabla III: Valor de la prueba de chi-cuadrado de Pearson para la asociación entre la frecuencia de utilización de la biblioteca de la UIB y bibliotecas no universitarias para buscar información con fines académicos y las características del alumnado

\begin{tabular}{|l|l|l|l|l|l|l|l|l|}
\cline { 2 - 10 } \multicolumn{1}{c|}{} & \multicolumn{4}{l}{ Biblioteca de la UIB } & \multicolumn{3}{c|}{ Bibliotecas no universitarias } \\
\hline Características de la muestra & $\mathbf{c}^{2}$ & $\mathbf{p}$ & $\mathbf{g . I}$ & $\mathbf{n}$ & $\mathbf{c}^{2}$ & $\mathbf{p}$ & g.I. & $\mathbf{n}$ \\
\hline Género & 6,276 & 0,043 & 2 & 720 & 2,644 & 0,267 & 2 & 710 \\
\hline Edad & 16,566 & 0,011 & 6 & 721 & 3,939 & 0,685 & 6 & 711 \\
\hline Curso matriculado & $46,129 *$ & 0,000 & 6 & 721 & 16,167 & 0,013 & 6 & 711 \\
\hline Área de conocimiento & $61,078^{*}$ & 0,000 & 6 & 721 & $41,043^{*}$ & 0,000 & 6 & 711 \\
\hline $\begin{array}{l}\text { Número de trabajos } \\
\text { elaborados por curso }\end{array}$ & 7,294 & 0,121 & 4 & 719 & 3,949 & 0,413 & 4 & 711 \\
$c^{2}$ Chi-cuadrado de Pearson // p Significación // g.l. Grados de libertad // * Significativa al nivel 0,01 (bilateral) \\
// n № de alumnado de la muestra
\end{tabular}

\subsubsection{Frecuencia de uso de la biblioteca de la UIB para buscar información con fines académicos y curso.}

Como refleja la gráfica siguiente, son los alumnos de últimos cursos -cuarto y quinto- los que hacen un uso más frecuente de la biblioteca de la UIB para buscar documentación académica; un $13,7 \%$ del alumnado de quinto curso y un $16,5 \%$ del alumnado de cuarto curso son usuarios habituales del servicio. En cursos inferiores, para el mismo perfil de usuario, el porcentaje baja hasta el $6 \%$ en tercero y el $3,5 \%$ en segundo curso. 


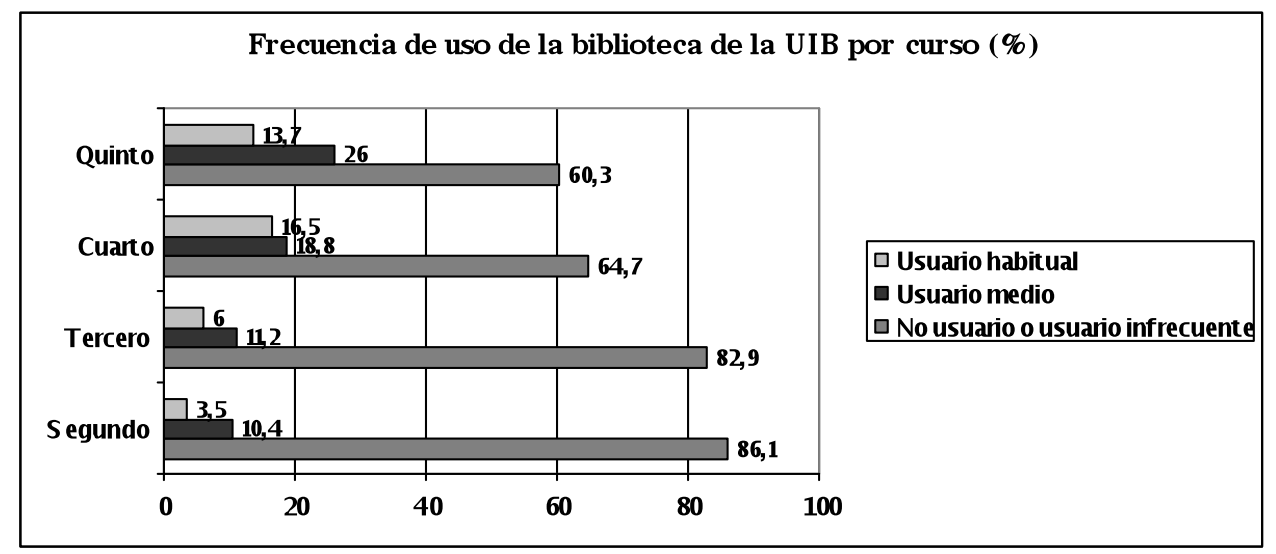

\subsubsection{Frecuencia de uso de la biblioteca de la UIB para buscar información con fines académicos y área de conocimiento.}

Son, con una diferencia muy significativa respecto al resto, los alumnos de Humanidades los que hacen un uso más extensivo de la biblioteca de la universidad: el 37,8\% manifiesta acudir habitualmente al catálogo de la UIB cuando tiene que buscar información para sus actividades académicas. Los menos proclives a emplear este tipo de servicio son los estudiantes de Ciencias Sociales y Jurídicas y Ciencias Experimentales.

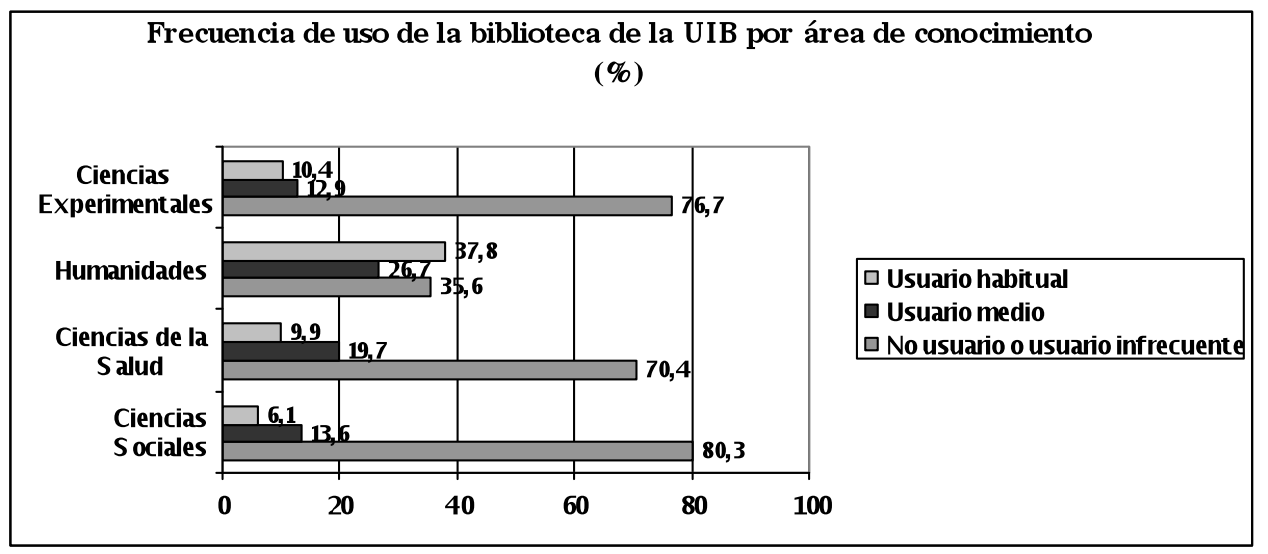

\subsubsection{Frecuencia de uso de bibliotecas no universitarias para buscar información con fines académicos y área de conocimiento.}

En la correlación entre el área de conocimiento y la frecuencia de uso de las bibliotecas no universitarias, vemos que, al igual que en el caso anterior, es el alumnado de Humanidades el que emplea con mayor frecuencia estos recursos para documentarse con fines académicos. 


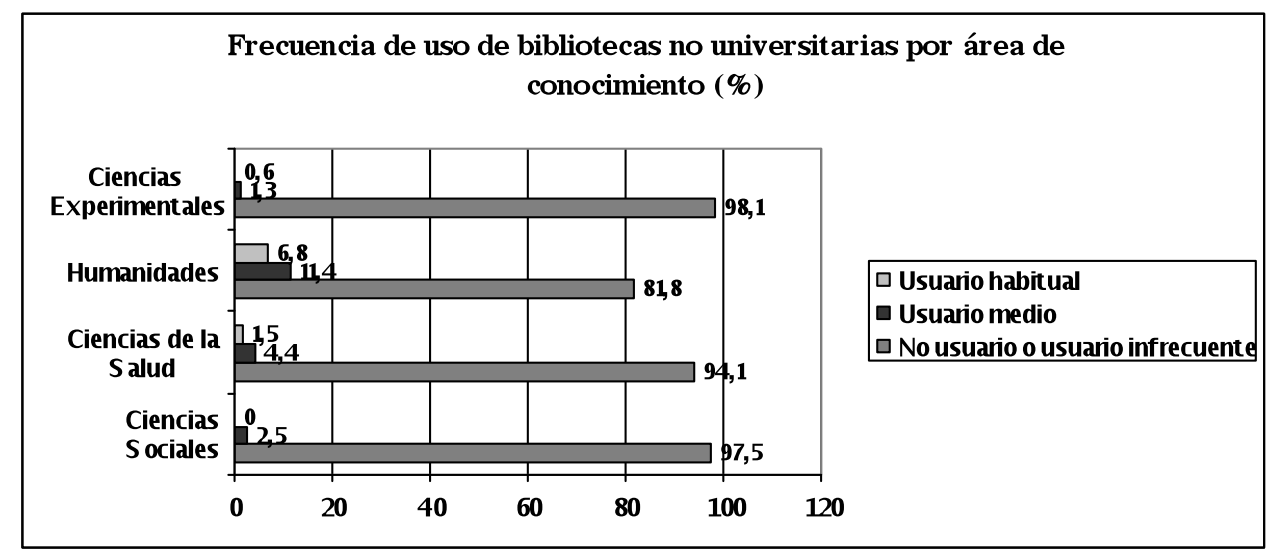

\subsection{Frecuencia de utilización de Internet (buscadores generalistas, portales especializados y bases de datos académicas) para buscar información con fines académicos.}

Los buscadores generalistas son los recursos de Internet que más frecuentemente emplea el alumnado para buscar información académica. Algo más del $70 \%$ de la muestra $(72,2 \%)$ manifiesta emplearlos siempre que necesita documentarse. En el polo opuesto se sitúa la frecuencia de uso de las bases de datos académicas: el 40,2\% afirma no utilizarlas nunca cuando tiene que buscar información. En un punto intermedio, aun cuando más cerca de la baja frecuencia de uso de las bases de datos académicas que de la alta frecuencia de utilización de los buscadores se coloca el empleo de portales especializados como fuentes documentales.

Al respecto de las correlaciones entre el uso de los dichos recursos comentados y las variables definitorias de la muestra conviene apuntar que: a) en cuanto al uso de buscadores generalistas: se da una asociación fuerte entre la frecuencia de uso de los mismos el género, la edad y el número de trabajos elaborados por curso; y b) en cuanto al uso de los portales especializados: existe significación entre la frecuencia de uso y el número de trabajos elaborados por curso. No se ha encontrado relación estadísticamente significativa entre la frecuencia de utilización de las bases de datos académicas y las características descriptivas de la muestra.

Tabla IV: Frecuencia de utilización de recursos de Internet para buscar información académica entre el alumnado a lo largo del curso 2008-09 ( $N=739)$

\begin{tabular}{|l|l|l|l|}
\hline $\begin{array}{l}\text { Frecuencia de } \\
\text { utilización }\end{array}$ & Buscadores & $\begin{array}{l}\text { Portales } \\
\text { especializados }\end{array}$ & $\begin{array}{l}\text { Bases de } \\
\text { datos } \\
\text { académicas }\end{array}$ \\
\hline Nunca & $1,4 \%$ & $21,1 \%$ & $40,2 \%$ \\
\hline A veces & $1,9 \%$ & $36,8 \%$ & $37,9 \%$ \\
\hline A menudo & $4,7 \%$ & $24,5 \%$ & $12,4 \%$ \\
\hline Muy a menudo & $18,8 \%$ & $11 \%$ & $3,7 \%$ \\
\hline Siempre & $72,7 \%$ & $2,7 \%$ & $1,2 \%$ \\
\hline No sabe o No & $0,5 \%$ & $3,9 \%$ & $4,6 \%$ \\
\hline
\end{tabular}




\begin{tabular}{|l|l|l|l|}
\hline contesta & & & \\
\hline Total & $100 \%$ & $100 \%$ & $100 \%$ \\
\hline
\end{tabular}

Tabla V: Valor de la prueba de chi-cuadrado de Pearson para la asociación entre la frecuencia de utilización de recursos de Internet para buscar información con fines académicos y las características del alumnado

\begin{tabular}{|c|c|c|c|c|c|c|c|c|c|c|c|c|}
\hline \multirow[b]{2}{*}{$\begin{array}{l}\text { Característica } \\
\text { s de } \\
\text { la muestra }\end{array}$} & \multicolumn{4}{|c|}{ Buscadores generalistas } & \multicolumn{4}{|c|}{ Portales especializados } & \multicolumn{4}{|c|}{$\begin{array}{l}\text { Bases de } \\
\text { académicas }\end{array}$} \\
\hline & $\mathrm{c}^{2}$ & p & g.I & $\mathrm{n}$ & $c^{2}$ & $p$ & $\begin{array}{l}\text { g.l } \\
.\end{array}$ & $\mathrm{n}$ & $c^{2}$ & $p$ & g.I & $\mathrm{n}$ \\
\hline Género & $\begin{array}{l}12,644 \\
*\end{array}$ & $\begin{array}{l}, 00 \\
2 \\
\end{array}$ & 2 & $\begin{array}{l}73 \\
4 \\
\end{array}$ & 1,486 & $\begin{array}{l}0,47 \\
6 \\
\end{array}$ & 2 & $\begin{array}{l}70 \\
9 \\
\end{array}$ & $\begin{array}{l}2,55 \\
7 \\
\end{array}$ & $\begin{array}{l}0,27 \\
8 \\
\end{array}$ & 2 & $\begin{array}{l}70 \\
4 \\
\end{array}$ \\
\hline Edad & $\begin{array}{l}33,957 \\
*\end{array}$ & $\begin{array}{l}0,01 \\
1\end{array}$ & 6 & $\begin{array}{l}73 \\
5\end{array}$ & 14,047 & $\begin{array}{l}0,02 \\
9\end{array}$ & 6 & $\begin{array}{l}71 \\
0\end{array}$ & $\begin{array}{l}6,03 \\
9\end{array}$ & $\begin{array}{l}0,41 \\
0\end{array}$ & 6 & $\begin{array}{l}70 \\
5\end{array}$ \\
\hline $\begin{array}{l}\text { Curso } \\
\text { matriculado }\end{array}$ & 3,662 & $\begin{array}{l}0,72 \\
2\end{array}$ & 6 & $\begin{array}{l}73 \\
5\end{array}$ & 7,167 & $\begin{array}{l}0,30 \\
6\end{array}$ & 6 & $\begin{array}{l}71 \\
0\end{array}$ & $\begin{array}{l}3,07 \\
4\end{array}$ & $\begin{array}{l}0,80 \\
3\end{array}$ & 6 & $\begin{array}{l}70 \\
5\end{array}$ \\
\hline $\begin{array}{l}\text { Área de } \\
\text { conocimiento }\end{array}$ & 7,822 & $\begin{array}{l}0,25 \\
1 \\
\end{array}$ & 6 & $\begin{array}{l}73 \\
5 \\
\end{array}$ & 5,572 & $\begin{array}{l}0,47 \\
3 \\
\end{array}$ & 6 & $\begin{array}{l}71 \\
0 \\
\end{array}$ & $\begin{array}{l}3,15 \\
1 \\
\end{array}$ & $\begin{array}{l}0,79 \\
0 \\
\end{array}$ & 6 & $\begin{array}{l}70 \\
5 \\
\end{array}$ \\
\hline $\begin{array}{l}\text { Número de } \\
\text { trabajos } \\
\text { elaborados } \\
\text { por curso }\end{array}$ & $\begin{array}{l}17,211 \\
*\end{array}$ & $\begin{array}{l}0,00 \\
2\end{array}$ & 4 & $\begin{array}{l}73 \\
3\end{array}$ & $\begin{array}{l}31,383 \\
*\end{array}$ & $\begin{array}{l}0,00 \\
2\end{array}$ & 4 & $\begin{array}{l}70 \\
9\end{array}$ & $\begin{array}{l}7,03 \\
1\end{array}$ & $\begin{array}{l}0,13 \\
4\end{array}$ & 4 & $\begin{array}{l}70 \\
3\end{array}$ \\
\hline
\end{tabular}

$\mathrm{c}^{2}$ Chi-cuadrado de Pearson // p Significación // g.l. Grados de libertad $/ / *$ Significativa al nivel 0,01 (bilateral) $/ / \mathrm{n}$ № de alumnado de la muestra

\subsubsection{Frecuencia de uso de motores de búsqueda para localizar información con fines académicos y género.}

Las alumnas muestran tasas de frecuencia más elevadas que los alumnos a la hora de usar los buscadores generalistas para localizar información de carácter académico. Como ilustra la gráfica siguiente, un $94,9 \%$ de las universitarias son usuarias habituales de estos recursos, mientras que entre ellos el porcentaje se sitúa en el $87,7 \%$. Siendo, de cualquier manera, en ambos casos, una tasa de frecuencia muy alta. 


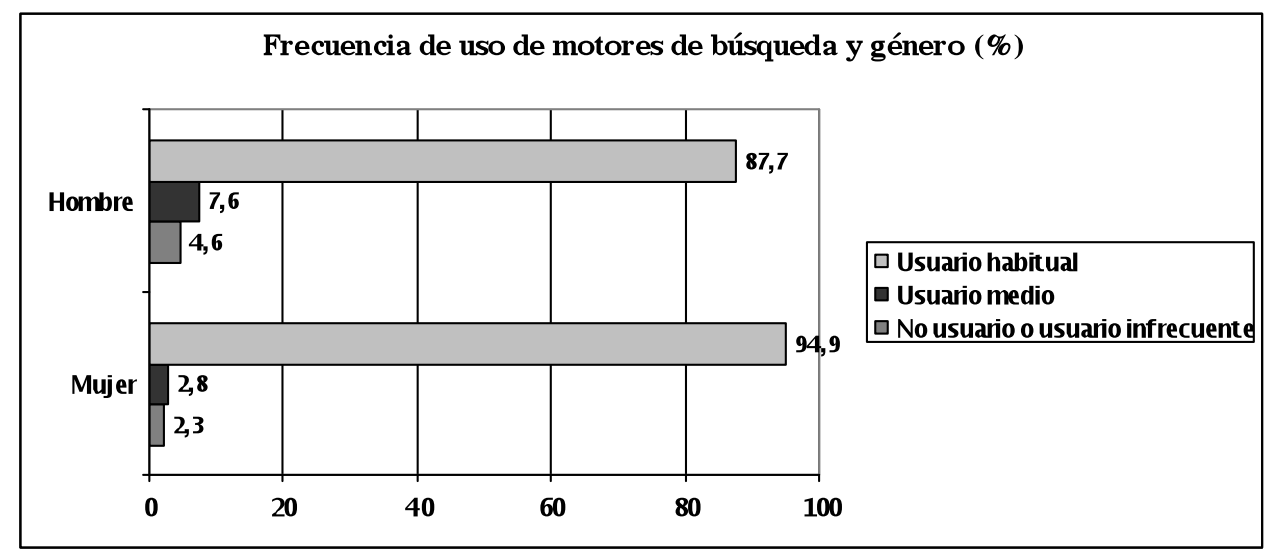

\subsubsection{Frecuencia de uso de motores de búsqueda para localizar información con fines académicos y edad.}

A mayor edad, parece darse un menor uso de los motores de búsqueda para encontrar información con fines académicos. Son los alumnos más jóvenes los que hacen un uso más frecuente de los buscadores $(95,2 \%$ del alumnado de entre 17 y 20 años se declaran usuarios habituales); mientras que aquellos con edades por encima de los treinta años manifiestan tasas de utilización algo más bajas (76\% se declara usuario habitual).

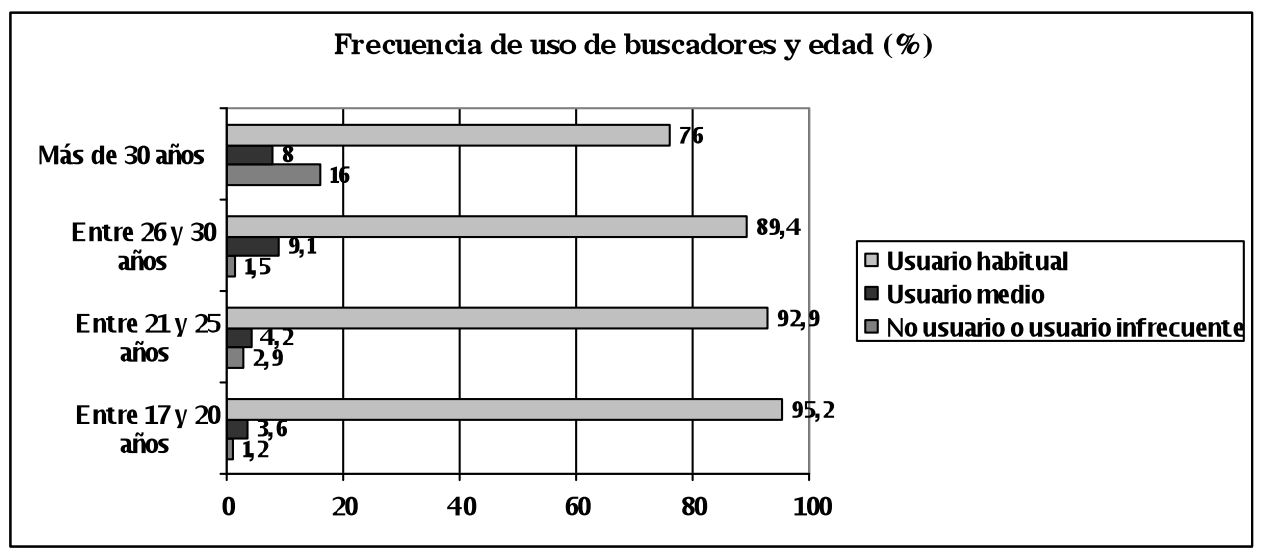

\subsubsection{Frecuencia de uso de motores de búsqueda para localizar información con fines académicos y número de trabajos elaborados.}

El alumnado que elabora más trabajos por curso es el que se muestra tasas de frecuencia más altas en cuanto al empleo de los buscadores generalistas. Así, el 96,6\% del alumnado que durante el curso académico 2008-2009 elaboró más de once trabajos es usuario habitual de los buscadores para localizar información. Entre los alumnos que realizaron entre ninguno y cinco trabajos, el porcentaje de usuarios habituales desciende hasta el $83,3 \%$. 


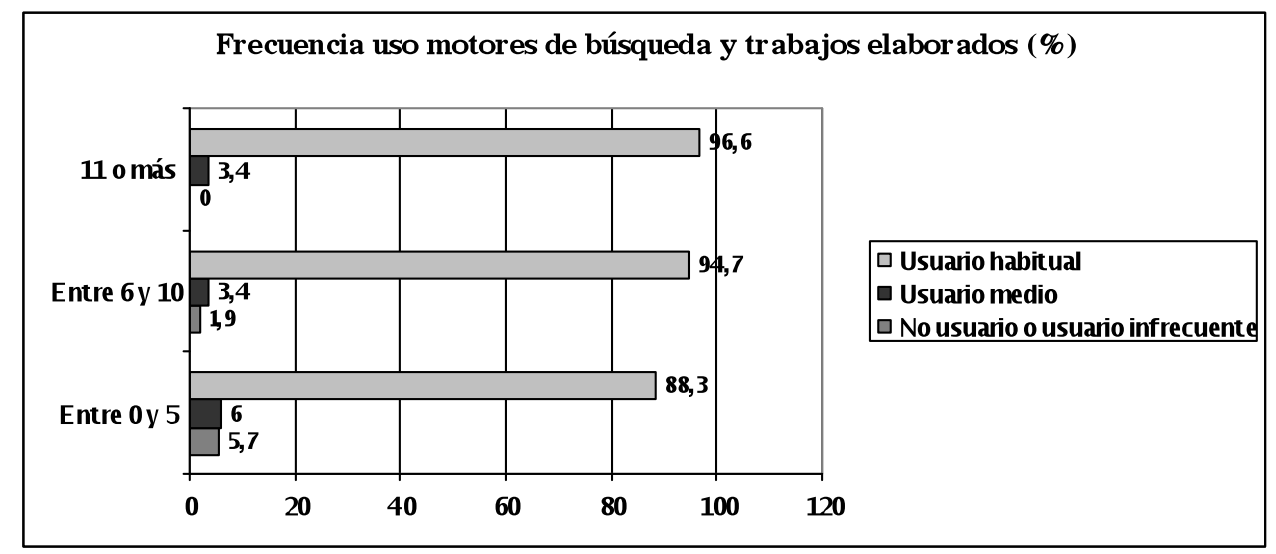

\subsubsection{Frecuencia de uso de portales especializados para localizar información con fines académicos y número de trabajos elaborados.}

Al igual que en el caso precedente, a mayor número de trabajos elaborados mayores tasas de frecuencia resultan. El 22,9\% del alumnado que entregó más de 11 trabajos es usuario habitual de los portales especializados para documentarse con fines académicos. Entre los alumnos que no entregaron ningún trabajo o entregaron un máximo de 5 , el porcentaje de usuarios infrecuentes o no usuarios es del $68,4 \%$.

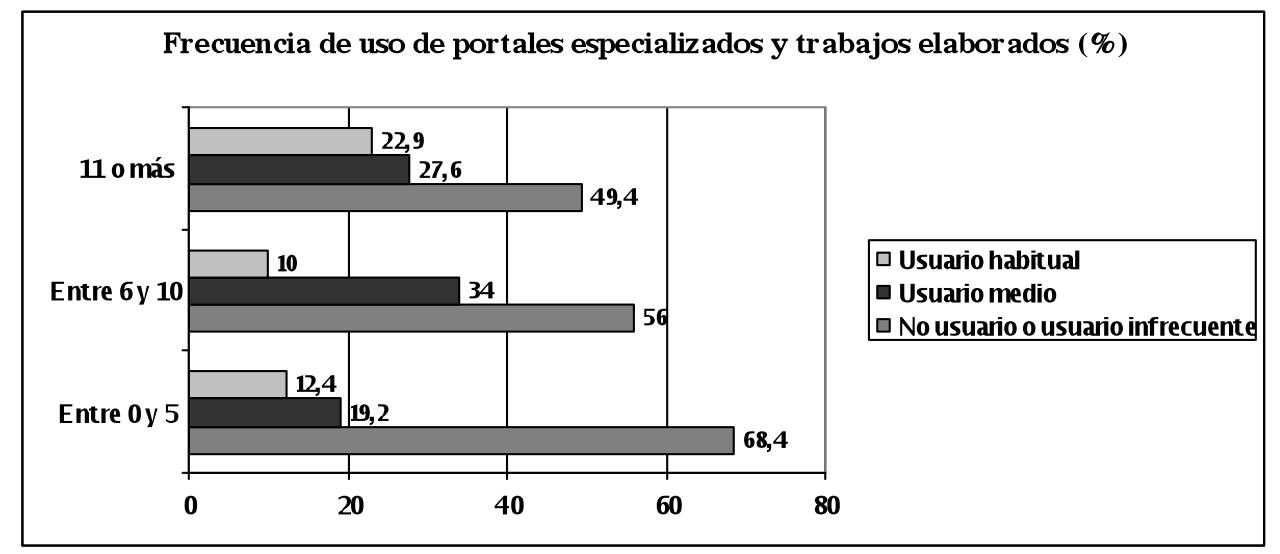

\subsection{Frecuencia de utilización de Internet para realizar actividades académicas.}

Internet es utilizado por el alumnado, principalmente, para elaborar trabajos académicos (véase Tabla VI). El 66,7\% de los encuestados manifiesta hacer uso de la Red para preparar trabajos "muy a menudo" o "siempre". La segunda de las actividades para la que el uso de la Red es más frecuente es para preparar o ampliar apuntes: el 26,5\% lo hace "muy a menudo" o "siempre". El $58,6 \%$ afirma no usar nunca Internet o usarla "a veces" para preparar exámenes.

En cuanto a la correlación de estas variables de frecuencia con las característica de la muestra, es significativo: a) en relación al uso de Internet para hacer trabajos: la existencia de relación fuerte entre la frecuencia de utilización y el curso y el área de conocimiento; b) respecto a la ampliación de apuntes: se constata una alta significación entre la frecuencia de uso de Internet y, 
nuevamente, el curso y el área de conocimiento; y c) en referencia a la preparación de exámenes: se da una fuerte relación entre la frecuencia de uso de Internet con este fin y el género y el área de conocimiento.

Tabla VI: Frecuencia de utilización de Internet para realizar actividades académicas entre el alumnado $(\mathrm{N}=739)$

\begin{tabular}{|l|l|l|l|}
\hline $\begin{array}{l}\text { Frecuencia de } \\
\text { utilización }\end{array}$ & $\begin{array}{l}\text { Elaborar } \\
\text { trabajos }\end{array}$ & $\begin{array}{l}\text { Ampliar } \\
\text { apuntes }\end{array}$ & $\begin{array}{l}\text { Preparar } \\
\text { exámenes }\end{array}$ \\
\hline Nunca & $1,9 \%$ & $10,4 \%$ & $17,6 \%$ \\
\hline A veces & $8,1 \%$ & $40,3 \%$ & $41 \%$ \\
\hline A menudo & $21,5 \%$ & $21 \%$ & $22,3 \%$ \\
\hline Muy a menudo & $33,4 \%$ & $18 \%$ & $11,1 \%$ \\
\hline Siempre & $33,3 \%$ & $8,5 \%$ & $5,8 \%$ \\
\hline $\begin{array}{l}\text { No sabe o No } \\
\text { contesta }\end{array}$ & $1,8 \%$ & $1,8 \%$ & $2,2 \%$ \\
\hline Total & $100 \%$ & $100 \%$ & $100 \%$ \\
\hline
\end{tabular}

Tabla V: Valor de la prueba de chi-cuadrado de Pearson para la asociación entre la frecuencia de utilización de Internet para realizar actividades académicas y las características del alumnado

\begin{tabular}{|c|c|c|c|c|c|c|c|c|c|c|c|c|}
\hline & \multicolumn{4}{|c|}{ Elaborar trabajos } & \multicolumn{4}{|c|}{ Ampliar apuntes } & \multicolumn{4}{|c|}{ Preparar exámenes } \\
\hline $\begin{array}{l}\text { Característica } \\
\text { s de } \\
\text { la muestra }\end{array}$ & $c^{2}$ & $p$ & $\begin{array}{l}\text { g.I } \\
.\end{array}$ & $\mathrm{n}$ & $c^{2}$ & $p$ & $\begin{array}{l}\text { g.I } \\
.\end{array}$ & $n$ & $c^{2}$ & $p$ & $\begin{array}{l}\text { g.I } \\
.\end{array}$ & $n$ \\
\hline Género & 3,352 & $\begin{array}{l}0,18 \\
7\end{array}$ & 2 & $\begin{array}{l}72 \\
5\end{array}$ & 1,074 & $\begin{array}{l}0,58 \\
4\end{array}$ & 2 & $\begin{array}{l}72 \\
5\end{array}$ & $\begin{array}{l}10,047 \\
*\end{array}$ & $\begin{array}{l}0,00 \\
7\end{array}$ & 2 & $\begin{array}{l}72 \\
4\end{array}$ \\
\hline Edad & 11,406 & $\begin{array}{l}0,07 \\
7\end{array}$ & 6 & $\begin{array}{l}72 \\
6 \\
\end{array}$ & 16,170 & $\begin{array}{l}0,01 \\
3\end{array}$ & 6 & $\begin{array}{l}72 \\
6\end{array}$ & 6,507 & $\begin{array}{l}0,36 \\
9\end{array}$ & 6 & $\begin{array}{l}72 \\
3\end{array}$ \\
\hline $\begin{array}{l}\text { Curso } \\
\text { matriculado }\end{array}$ & $\begin{array}{l}18,608 \\
*\end{array}$ & $\begin{array}{l}0,00 \\
5\end{array}$ & 6 & $\begin{array}{l}72 \\
6 \\
\end{array}$ & $\begin{array}{l}24,889 \\
*\end{array}$ & $\begin{array}{l}0,00 \\
0\end{array}$ & 6 & $\begin{array}{l}72 \\
6\end{array}$ & 9,621 & $\begin{array}{l}0,00 \\
0\end{array}$ & 6 & $\begin{array}{l}72 \\
6\end{array}$ \\
\hline $\begin{array}{l}\text { Área de } \\
\text { conocimiento }\end{array}$ & $\begin{array}{l}18,859 \\
*\end{array}$ & $\begin{array}{l}0,00 \\
4 \\
\end{array}$ & 6 & $\begin{array}{l}72 \\
6 \\
\end{array}$ & $\begin{array}{l}25,415 \\
*\end{array}$ & $\begin{array}{l}0,00 \\
0 \\
\end{array}$ & 6 & $\begin{array}{l}72 \\
6 \\
\end{array}$ & $\begin{array}{l}30,273 \\
*\end{array}$ & $\begin{array}{l}0,14 \\
2 \\
\end{array}$ & 6 & $\begin{array}{l}72 \\
3 \\
\end{array}$ \\
\hline
\end{tabular}

$c^{2}$ Chi-cuadrado de Pearson // p Significación // g.l. Grados de libertad // * Significativa al nivel 0,01 (bilateral) // $\mathrm{n}$ № de alumnado de la muestra

\subsubsection{Frecuencia de utilización de Internet para elaborar trabajos académicos y curso.}

Se da la circunstancia que los alumnos de los cursos superiores son los que muestran tasas de frecuencia de utilización de Internet para hacer trabajos más altas. Así, el 79\% del alumnado de quinto curso es usuario de la Red cuando debe preparar trabajos, un $72,3 \%$ del de cuarto, un $66,4 \%$ del de tercero y un $59,4 \%$ del de segundo. 


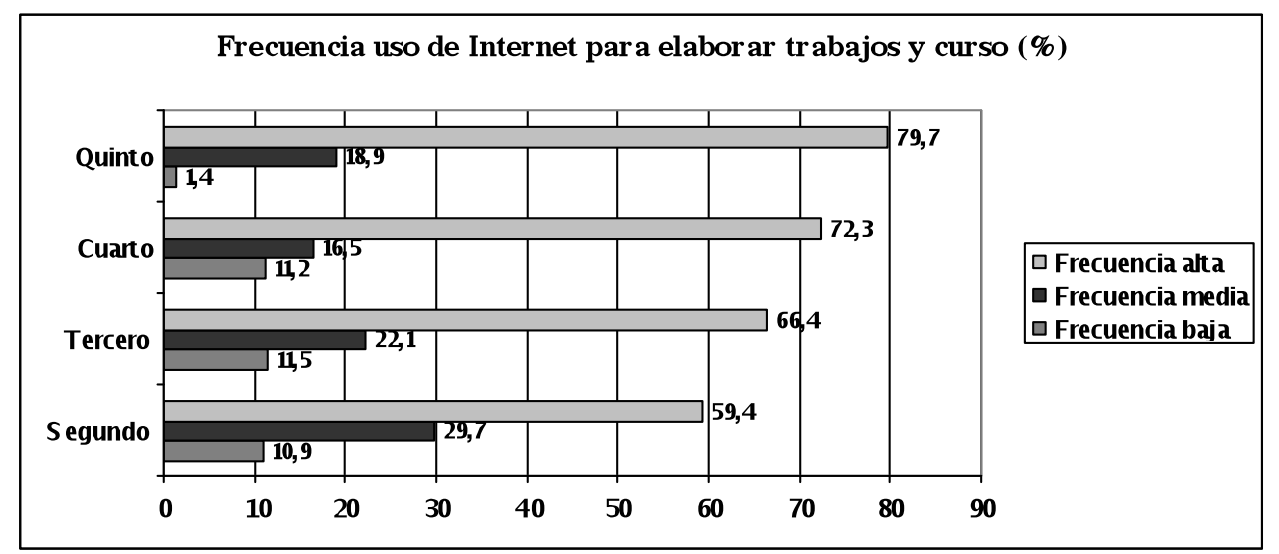

\subsubsection{Frecuencia de utilización de Internet para elaborar trabajos académicos y área de conocimiento.}

El alumnado de titulaciones del área de Ciencias Experimentales es el que hace un uso más frecuente de la Red para elaborar trabajos académicos: el 73,9\% presenta tasas de frecuencia alta. En segundo lugar se sitúan los alumnos de Ciencias Sociales. Los alumnos que menos utilizan Internet para elaborar trabajos son los de Humanidades: un 55,5\% presentan tasas de frecuencia alta y un $26,7 \%$ manifiestan tasas de frecuencia baja.

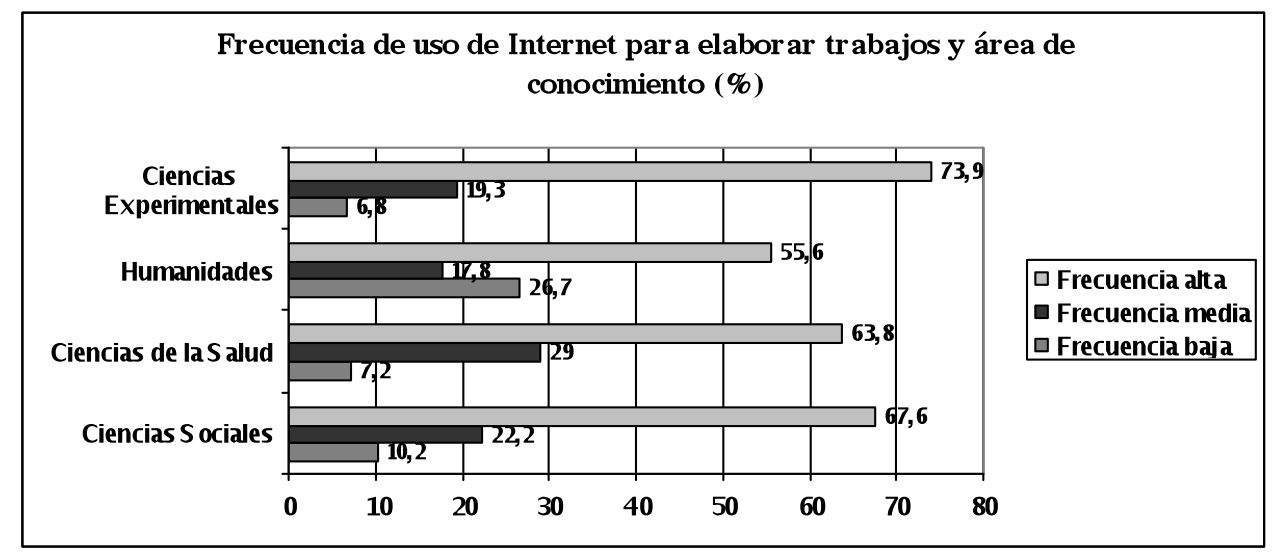

\subsubsection{Frecuencia de utilización de Internet para ampliar apuntes y curso.}

Nuevamente, el alumnado de los cursos superiores es el que demuestra hacer un uso más frecuente de la Red para, en este caso, preparar o ampliar apuntes de clase. El 37,8\% de los alumnos de quinto emplea Internet para ampliar apuntes, el 33,6\% de cuarto, el $22,8 \%$ de tercero y el $20 \%$ de segundo. 


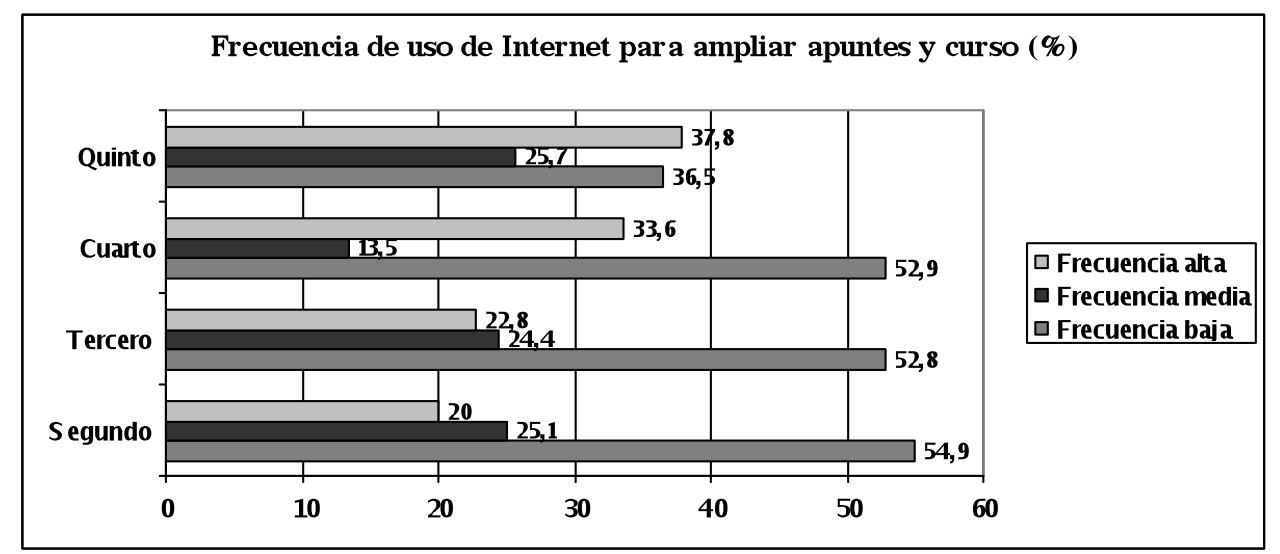

\subsubsection{Frecuencia de utilización de Internet para ampliar apuntes y área de conocimiento.}

El alumnado de Ciencias Experimentales es el que más usa Internet para ampliar o preparar apuntes: el 40,7\% presenta una frecuencia alta en esta cuestión. Los que menos emplean los recursos propios de la Red son los alumnos de Ciencias Sociales y Jurídicas: el 55,6\% manifiesta un perfil de frecuencia bajo.

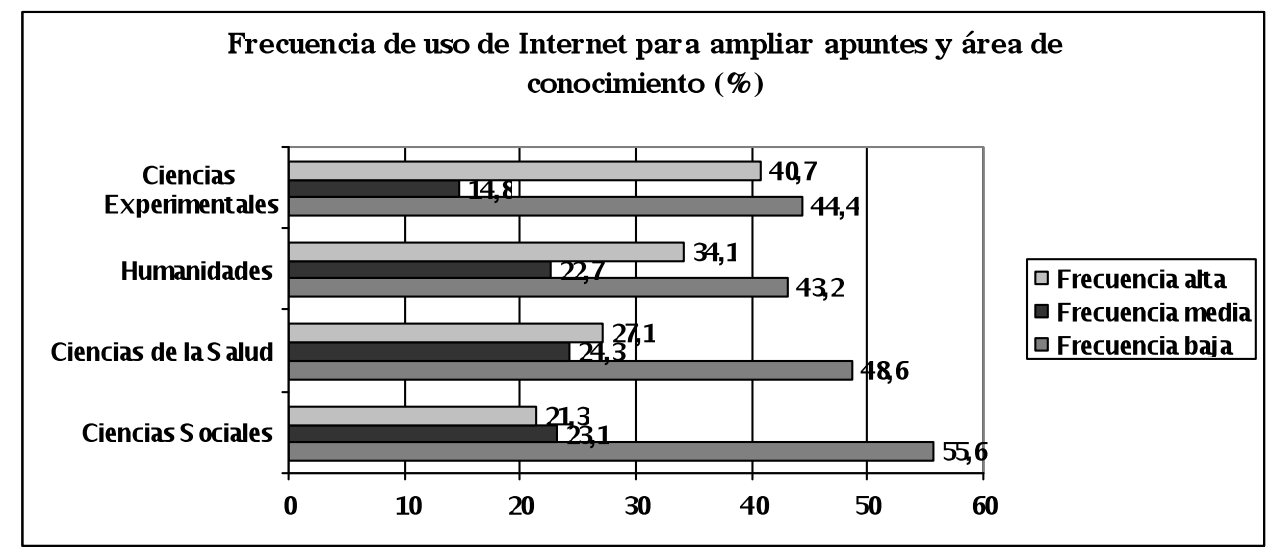

\subsubsection{Frecuencia de utilización de Internet para preparar exámenes y género.}

Los alumnos utilizan con mayor frecuencia Internet para prepararse exámenes que las alumnas. El $64,5 \%$ de ellas presenta tasas de frecuencia bajas en esta cuestión; mientras que en ellos, para el mismo rango, el porcentaje se sitúa en el 53,5\%. 


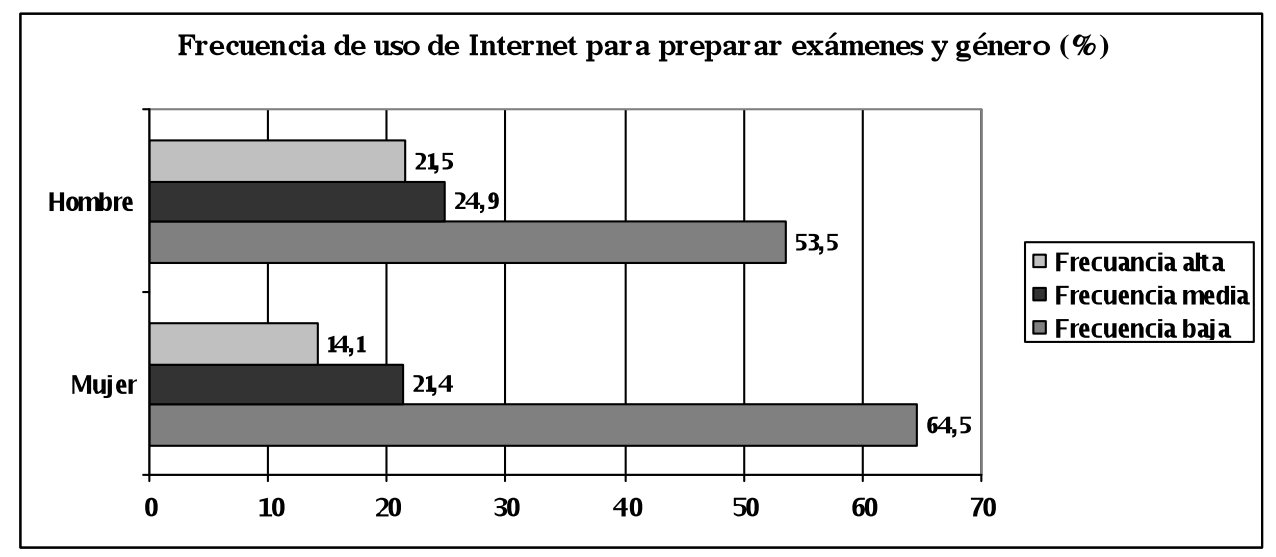

\subsubsection{Frecuencia de utilización de Internet para preparar exámenes y área de conocimiento.}

Tal y como ha venido dándose en cuestiones precedentes, los alumnos de Ciencias Experimentales son los que más usan la Red para preparar exámenes: casi el 30\% presenta frecuencias altas en esta cuestión. En segundo lugar se sitúan, en orden decreciente, los alumnos de Humanidades: un 20,9\% presenta tasas de frecuencia altas. Seguidamente se colocaría el alumnado de Ciencias de la Salud: un 17,1\% presenta perfiles de uso altos. En último lugar se sitúan los alumnos de Ciencias Sociales y jurídicas: un 12,7\% manifiesta frecuencias altas de utilización de Internet para preparar exámenes.

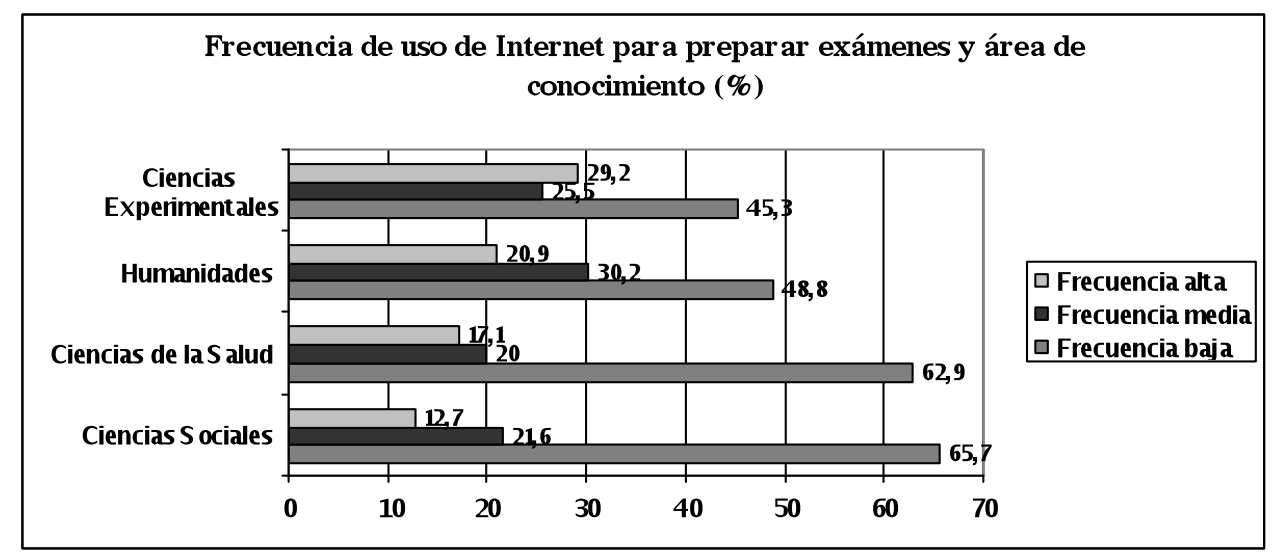

\section{CONCLUSIONES Y DISCUSIÓN.}

A tenor de los resultados alcanzados en el presente estudio, se puede argumentar, como primera de las conclusiones, que Internet monopoliza las búsquedas de información con fines académicos entre el alumnado universitario. Las tasas de frecuencia de uso para buscar información en las bibliotecas no universitarias son realmente bajas y son, de las tres fuentes documentales estudiadas, las menos empleadas por el alumnado cuando intentan localizar información con fines académicos. En cuanto a las tasas de uso de la biblioteca de la UIB como fuente documental, apuntar que: aun presentando niveles superiores a los descritos en el caso de las bibliotecas no universitarias se sitúa a una relevante distancia del uso de Internet como fuente de información académica. En cuanto al uso de las bibliotecas, repasando los resultados en función de las características del alumnado es preciso incidir en el hecho que el alumnado que utiliza con mayor 
frecuencia estos recursos es el que cursa estudios de últimos años (cuarto y sobre todo quinto) en carreras del área de Humanidades; mientras que los alumnos de cursos inferiores y de estudios de Ciencias Experimentales y Ciencias Sociales y Jurídicas son los que menos frecuencia de uso demuestran.

Ya centrándonos en el análisis del uso de Internet como fuente documental, es interesante constatar como el principal recurso empleado por el alumnado son los buscadores generalistas, bastante por encima de otros recursos como son los portales especializados y las bases de datos académicas. Es destacable el hecho que las mujeres empleen con mayor frecuencia que los hombres estos recursos, así como que a menor edad más frecuencia de uso y a mayor número de trabajos elaborados por curso mayores tasas de utilización presentan.

De las posibles actividades académicas para las que se puede hacer uso de Internet como fuente de información, la elaboración de trabajos es la más recurrente entre el alumnado de la UIB. Parece que la Red no juega un papel tan importante cuando los universitarios deben preparar o ampliar apuntes y preparar exámenes. Los que más utilizan Internet para elaborar trabajos y preparar apuntes son los alumnos de últimos cursos de carreras de Ciencias Experimentales, mientras que los menos adeptos a la Red para el desarrollo de dicha actividad serían los alumnos de Humanidades. En cuanto a la preparación de exámenes mediante el uso de Internet: los alumnos presentan tasas de frecuencia superiores que las alumnas $y$, nuevamente, es el alumnado de Ciencias Experimentales el que más emplea este recurso con dicha finalidad.

Internet, tal y como subrayan y apoyan numerosos estudios (Sureda y Comas, 2006), es la principal fuente de documentación del alumnado y ha provocado con su uso extensivo que las bibliotecas hayan visto reducida su finalidad como centros de documentación a centros de trabajo y estudio. Y ello quizás se haya visto todavía más agravado con la reciente aplicación del Espacio Europeo de Educación Superior y los planteamientos pedagógico-metodológicos que emanan del mismo; el tiempo dirá si eso es así y hasta qué punto, todavía es pronto para establecer posiciones a ese respecto. La cohorte generacional que transita por nuestras aulas universitarias presenta una serie de características definitorias ampliamente comentadas en la literatura al respecto y que tomaron forma, sobre todo, a partir del concepto acuñado por Prensky (2001) de "nativos digitales". Ahora bien, el hecho de su identidad o "alumbramiento" digital no puede hacernos caer en el error de considerarlos competentes de facto en el uso y manejo de las TIC. Y menos aún aventurar que el acceso y uso extensivo de estos medios y recursos con fines académicos asegura unos mejores resultados en la formación de los alumnos. Debemos, a este respecto, separarnos de lecturas que buscan equiparar acceso con competencia. Conviene asegurarnos, una vez comprobado que el acceso está garantizado y plenamente consolidado su uso, el empleo competente de las TIC con fines académicos. El dato aportado por nuestro trabajo, que deja bien a las claras el uso que hacen de los buscadores como principal fuente documental, muy por encima de otros recursos con mayor valor académico como puedan ser los portales especializados o las bases de datos, nos lleva a pensar en estrategias de búsqueda documental poco rigurosas, de escaso valor académico y escaso bagaje formativo. En este sentido, son necesarias estrategias formativas, a nuestro entender, que desgooglicen la educación y abran ventanas al conocimiento a partir del uso y aplicación de recursos y medios más pertinentes para favorecer que nuestros nativos digitales se puedan convertir en verdaderos ciudadanos digitales. 


\section{REFERENCIAS.}

ACRL/ALA (2000): Information Literacy Competency Standards for Higher Education. Disponible en http://www.ala.org/acrl/ilcomstan.html [Consulta: 2 de enero de 2010].

Aditaka, A. A. y Anwar, M. (2006). Student library use: a study of faculty perceptions in a Malaysian university. Librabry review, Vol. 55, no 2, pp. 106-119.

Agosto, D., Paone, K. y Ipock, G. (2007). The Female-Friendly Public Library: Gender Differences in Adolescents' Uses and Perceptions of U.S. Public Libraries. Library Trends, Vol. 56, no 2, pp. 387401.

Arias, A. y Simón, J. (2008). Estudio de los hábitos de uso de los estudiantes en la biblioteca de la Universidad Complutense de Madrid. Revista Española de Documentación Científica, Vol. 31, № 3, pp. 413-431.

Baer, W. y Li, L. (2009, Junio). Library and information use patterns by engineering faculty and students. ASEE Annual Conference 2009, Austin, TX. Consultado el 05-12-2009 de: https://smartech.gatech.edu/bitstream/1853/28688/1/EngLibSurvey-Bear-Li.pdf

Bawden, D. (2000). Revisión de los conceptos de alfabetización informacional y alfabetización digital. Anales de Documentación, no 5, pp. 361-408. ISSN: 1575-2437.

Bridges, J. (2008). Who Is Not Using the Library? A Comparison of Undergraduate Academic Disciplines and Library Use. Libraries and the Academy, Vol. 8, no 2, pp. 187-196.

Coll, C. y Rodríguez, J. (2008). Alfabetización, nuevas alfabetizaciones y alfabetización digital: las TIC en el currículum escolar. En Coll, C. yMonereo, C. (Eds.), Psicología de la educación virtual (pp. 325-347). Madrid: Morata.

Comas, R. (2009). El cibeplagio académico entre el alumnado universitario. Tesis Doctoral no publicada, Universidad de las Islas Baleares, España.

Hayden, H., O'Brien, T. y Rathaille, M. (2005). User Surrey at Waterford Institute of Technology Libraries. College Student Journal, Vol. 24, no 4, pp. 557-570.

Head, A. (2007). Beyond Google: how do students conducst academic research. First Monday, Vol. 12, $\quad n$ o $8 . \quad$ Consultado el 10-01-2010 de: http://firstmonday.org/htbin/cgiwrap/bin/ojs/index.php/fm/article/view/1998/1873

Hiller, S. y Self, J. (2002). A decade of user surveys: utilizing and accessing a standart assessment tool to measure library perfomance at the university of Virginia and university of Washington. Proceedings of the 4th Northumbria International Conference on Performance measures in libraries and Information services. 12-16 Agosto 2001. ARL Washington DC. Pp. 253-261.

Kuruppu, P. (2008). Information Use by PhD Students in Agriculture and Biology: A Dissertation Citation Analysis portal. Libraries and the Academy - Vol. 8, no 4, pp. 387-405.

Martin et al. (2009). Closing the Gap: Investigating the Search Skills of International and US Students: An Exploratory Study. Library Philosophy and Practice, Vol. Anual 2009. Consultado el 02-12-2009 de: http://www.webpages.uidaho.edu/ mbolin/lpp2009.htm 
Marzal, M. y Calzada, F. (2003). Un análisis de necesidades y hábitos informativos de estudiantes universitarios en Internet. Binaria: Revista de comunicación, cultura y tecnología, no. 3.

McKenna, J. (2007). Danish post-secondary students use public libraries for study purposes. Evidence based libary and information practice, Vol.2, no 3 .

McMartin et al (2008). The use of online digital resources and educational digital libraries in higher education. International Journal of digital libraries, Vol. 9, no 1, pp. 65-79.

Nwagwu, E. et. al (2009). Factors influencing use of the internet: A questionnaire survey of the students of University of Ibadan, Nigeria. The Electronic library, Vol. 27, no 4, pp. 718-734.

O'Hanlon, N. (2002). Net knowledge: performance of new college students on an Internet skills proficiency test. Internet and Higher Education, vol. 5, n. 1, pp. 55-66.

Prensky, M. (2001). Digital Natives, Digital Immigrants. On the Horizon, Vol. 9, no 5. Consultado el 12-12-2009

de:

www.marcprensky.com/.../Prensky\%20\%20Digital\%20Natives,\%20Digital\%20Immigrants\%20-

\%20Part1.pdf

Slaouti, D. (2002). The World Wide Web for academic purposes: old study skills for new?. English for Specific Purposes, vol. 21, n. 2, pp. 105-124.

Sureda, J. y Comas, R. (2006). Internet como fuente de documentación académica entre estudiantes universitarios. Palma de Mallorca: Fundación Universidad Empresa de las Islas Baleares. ISBN: 84-690-1270-3.

Varis, T. (2003). Nuevas formas de alfabetización y nuevas competencias en el e-learning. Documento electrónico en línea. Consultado (15-12-2009) en: http://www.elearningeuropa.info/directory/index.php?page $=$ doc \&doc id=595\&doclng $=7$

Vondracek, R. (2007). Comfort and Convenience? Why Students Choose Alternatives to the Library portal. Libraries and the Academy, Vol.7, no 3, pp. 277-293.

Wen-Hua, R. (2000). Library instruction and college student self-efficacy in electronic information searching. The Journal of Academic Librarianship, Vol. 26, n. 5, pp. 323-328.

Zhang, X., Anghelescu, H. y Yuan, X. (2005). Domain knowledge, search behaviour, and search effectiveness of engineering students: an exploratory study. Information Research, Vol. 10, no 2 . Consultado (15-12-2009) en: http://informationr.net/ir/10-2/paper217.html

\section{Para citar este artículo:}

COMAS, R., SUREDA, J. y MUT, T. (2010) « Uso de fuentes documentales -impresas y digitalescon fines académicos entre el alumnado universitario» [artículo en línea]. Edutec-e, Revista Electrónica de Tecnología Educativa. Núm. 31 / Febrero 2010. [Fecha de consulta: dd/mm/aa]. http://edutec.rediris.es/revelec2/revelec31/

ISSN 1135-9250. 